\title{
Microstates of systems obeying Maxwell-Boltzmann distribution, a limiting case of Quantum Statistics!
}

\author{
Sarmistha Sahu ${ }^{\# 1}$, M A Rama ${ }^{\# 2}$ \\ Department of Physics ${ }^{1}$, Department of ComputerScience ${ }^{2}$, Maharani Lakshmi Ammanni College for Women, \\ Bangalore 560012, India
}

\begin{abstract}
Thermodynamic probability $\Omega$ is defined as the number of alternative microscopic arrangements which correspond to the same macroscopic state. Thermodynamic probability $\Omega$ enables us to decide how much more probable certain situations are than others.

The method of determining the number of microstates for a given macrostate decides the statistics, BoseEinstein or Fermi-Dirac statistics; quantum or classical! The distribution of the particles in different microstates is demonstrated through animation with the help of computer graphics.
\end{abstract}

Keywords: Thermodynamic probability, microstates, macrostates, indistinguishability, energy level, degenerate states, Fermions, Bosons.

\section{Introduction}

The method of handling the extremely large thermodynamic probabilities encountered in real systems was first suggested in 1877 by the Austrian physicist Ludwig Boltzmann (1844 to 1906) through the equation $\mathrm{S}=\mathrm{k} \ln \Omega$ where $\mathrm{S}$ stands for entropy [1].

For real samples of matter, the values of $\Omega$ are of the order of $10^{1024}$ - so large that they are difficult to manipulate. Why and how this number is calculated, what it means, why a particular method is chosen to determine different systems and hence three different distributions can be explained with a computational model.

Turbo $\mathrm{C}$ with graphic functions is the language used for the animation. DosBox, a tool to demonstrate animation in full screen has been made use of.

\section{Distinguishable versus indistinguishable particles}

If we can track the trajectory of an identical particle, they are distinguishable, otherwise indistinguishable. Quantum particles with their waves overlapping are indiscernible, hence indistinguishable. Low density classical systems are identical but distinct.

Classical particle have undefined spins and wave functions. For low density systems, occupation number is one per cell and the particles are distinguishable described by Maxwell-Boltzmann statistics. But for higher density, any number of particles can occupy a cell.

\section{A. Exchange Symmetry}

For a two particle system, with states $n_{1}$ and $n_{2}$, the exchange symmetry of identical particles are related to spin and is given by $\left|n_{1} n_{2} \pm n_{2} n_{1}\right\rangle$, positive for symmetric and negative for asymmetric particles. The difference between the statistical behaviour of fermions, bosons and distinguishable particles for a two particle system is decided by the number of states and therefore the probability [2].

1) Two Identical distinguishable particles: Two identical distinguishable particles have four distinct states $|0\rangle|0\rangle ;|1\rangle|1\rangle ; \quad|0\rangle|1\rangle$ or $|1\rangle|0\rangle$ with probability of $25 \%, 25 \%$ and $50 \%$ respectively.

2) Identical indistinguishable particles with integral spin: Such particles have

$$
|0\rangle|0\rangle ;|1\rangle|1\rangle ; \frac{1}{\sqrt{2}}[|0\rangle|1\rangle+|1\rangle|0\rangle] \text { with probability of } 33 \% \text { each. }
$$

3) Identical indistinguishable particles with half integral spin: These have $\frac{1}{\sqrt{2}}[|0\rangle|1\rangle-|1\rangle|0\rangle]$ state with probability of $100 \%$. Pauli's exclusion principle does not permit the states $|0\rangle|0\rangle$ and $|1\rangle|1\rangle$.

Comparison of (1) and (2) suggests that the identical indistinguishable particles (bosons) can clump in a state which indicates Bose-Einstein Condensation. 


\section{Thermodynamic Potential}

Maxwell-Boltzmann distribution of $\mathrm{N}$ identical distinguishable particles in $\mathrm{g}$ states is $\Omega=g(g-1)(g-2) \ldots \ldots(g-\overline{N-1})$ but when the $g$ states are of same energy, the proximity of the states suggests that the particles can be indistinguishable! Then the number of microstates is given by $\Omega=g(g-1)(g-2) \ldots . . .(g-\overline{N-1}) / N$ !

The number of microstates of fermions (quantum particle, indistinguishable with half integral spin) is also given by $\Omega=g(g-1)(g-2) \ldots . . .(g-\overline{N-1}) / N$ !

No wonder, the Maxwell-Boltzmann statistics is a limiting case of Quantum Statistics!

Some of these facts are demonstrated by the animation of balls (particles) in cups (states). Distinguishable balls are differently coloured while indistinguishable balls are similar colour. States in different energy levels are stacked vertically while states in the same energy levels are in the horizontal line.

\section{A. Example One}

If there are 3 states and two balls, how many microstates exist for this macrostate depends on the distinguishability and the number of particles that can be accommodated in a state as shown Table 1.

1) One distinguishable particle per cell: For two distinguishable particles, arranging themselves in three states, occupying one per cell, the number of microstates is $\mathbf{\Omega}=\mathbf{g}(\mathbf{g}-\mathbf{1})(\mathbf{g}-\mathbf{2}) \ldots . . .(\mathbf{g}-(\mathbf{N}-\mathbf{1}))=\mathbf{3} \mathbf{2} \mathbf{2}=\mathbf{6}$ and leads to Maxwell-Boltzmann statistics when $\mathrm{N} \approx \mathrm{g}$. Refer to Table 1.

TABLE 1

One Distinguishable Particle Per Cell

\begin{tabular}{|l|l|l|l|l|l|}
\hline Yellow & Yellow & & Green & Green & \\
\hline Green & & Yellow & Yellow & & Green \\
\hline & Green & Green & & Yellow & Yellow \\
\hline
\end{tabular}

But when the particles become indistinguishable, it gives only 3 microstates leading to Fermi-Dirac statistics as shown in Table 4.

2) Any number of distinguishable particles per cell: On the other hand, for two distinguishable particles, arranging themselves in three states, with any number of particles occupying a cell the number of microstates is $\boldsymbol{\Omega}=\mathbf{g}^{\mathbf{N}}=\mathbf{3}^{\mathbf{2}}=\mathbf{9}$ and leads to Maxwell -Boltzmann statistics when $\mathrm{N} \gg \mathrm{g}$ as shown in Table 2.

But when the particles become indistinguishable, it gives only 6 microstates leading to Bose-Einstein Statistics. Then the number of microstates is given by $\Omega=(\mathrm{N}+\mathrm{g}-1) ! / \mathrm{N} !(\mathrm{g}-1) !=4 ! / 2 ! 2 !=6$ as shown in Table 3.

TABLE 2

ANY NUMBER OF DISTINGUISHABLE PARTICLES PER CELL

\begin{tabular}{|l|l|l|l|l|l|l|l|l|}
\hline Yellow & & $\begin{array}{l}\text { Pink } \\
\text { Yellow }\end{array}$ & & Yellow & & Pink & Pink & \\
\hline Pink & $\begin{array}{l}\text { Pink } \\
\text { Yellow }\end{array}$ & & & & Yellow & Yellow & & Pink \\
\hline & & & $\begin{array}{l}\text { Pink } \\
\text { Yellow }\end{array}$ & Pink & Pink & & Yellow & Yellow \\
\hline
\end{tabular}

TABLE 3

ANY NUMBER OF INDISTINGUISHABLE PARTICLES PER CELL

\begin{tabular}{|l|l|l|l|l|l|}
\hline Pink & & $\begin{array}{l}\text { Pink } \\
\text { Pink }\end{array}$ & & Pink & \\
\hline Pink & $\begin{array}{l}\text { Pink } \\
\text { Pink }\end{array}$ & & & & Pink \\
\hline & & & $\begin{array}{l}\text { Pink } \\
\text { Pink }\end{array}$ & Pink & Pink \\
\hline
\end{tabular}


3) Only one indistinguishable particle per cell: For two indistinguishable particles, arranging themselves in three states, with only one particle per cell the number of microstates is $\mathbf{\Omega}=\mathbf{g}(\mathbf{g}-\mathbf{1})(\mathbf{g}-$ 2)....(g-(N-1))/N!= 3x2/2! $=\mathbf{3}$ as shown in Table 4. This gives the Fermi-Dirac statistics.

TABLE 4

ONLY ONE INDISTINGUISHABLE PARTICLE PER CELL

\begin{tabular}{|c|c|c|}
\hline Pink & Pink & \\
\hline Pink & & Pink \\
\hline & Pink & Pink \\
\hline
\end{tabular}

\section{B. Example Two}

Let A, B, C be three particles in four states. Here we are showing schematically the different ways the particles can occupy the states, (1) when the states belong to different energy level and only one particle occupies a cell. (2) when the states belong to the same energy levels and one particle occupies a cell.

1) One distinguishable particle per cell, each state is in a different (vertical) energy level:

TABLE 5

ONE DISTINGUISHABLE PARTICLE PER CELL IN DIFFERENT ENERGY LEVELS

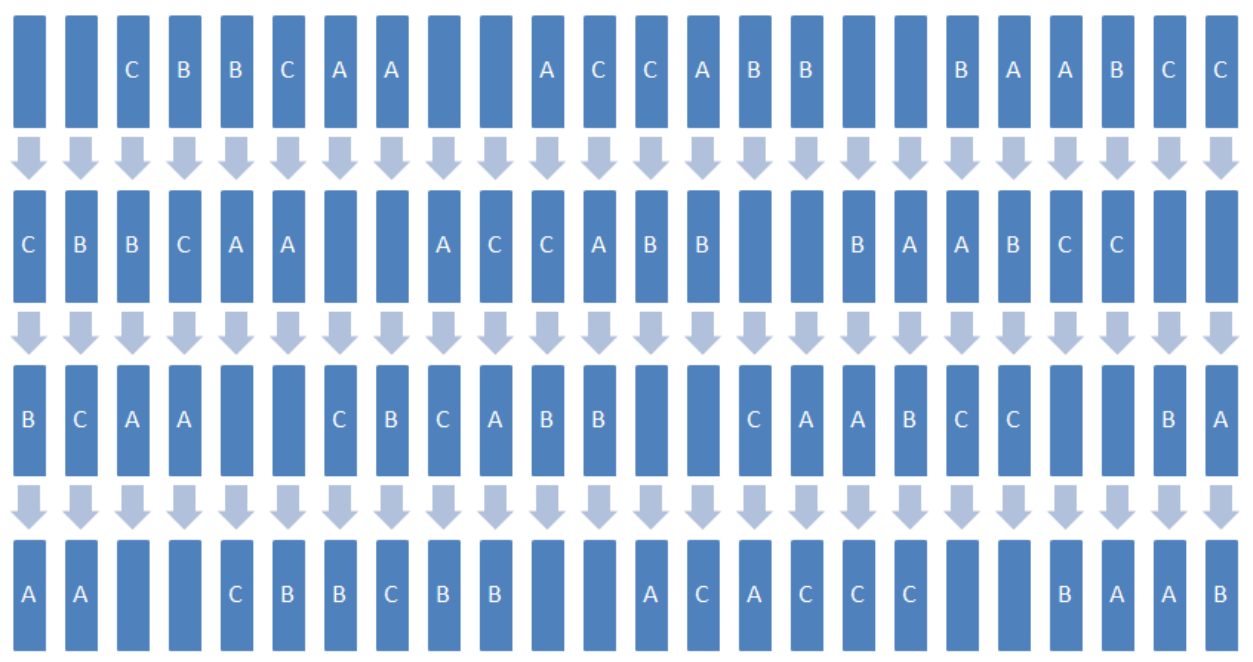

The number of microstates is

$\mathbf{\Omega}=\mathbf{g}(\mathbf{g}-\mathbf{1})(\mathbf{g}-\mathbf{2}) \ldots . .(\mathbf{g}-(\mathbf{N}-\mathbf{1}))=\mathbf{4} * \mathbf{3} * \mathbf{2}=\mathbf{2 4}$ as shown in the Table 5 .

2) One indistinguishable particle per cell, in degenerate energy states: When the states belong to the same energy level (horizontal), the particles become indistinguishable. The number of microstates for the given macrostate is shown in Table 6.

TABLE 6

ONE INDISTINGUISHABLE PARTICLE PER CELL IN DEGENERATE ENERGY STATES

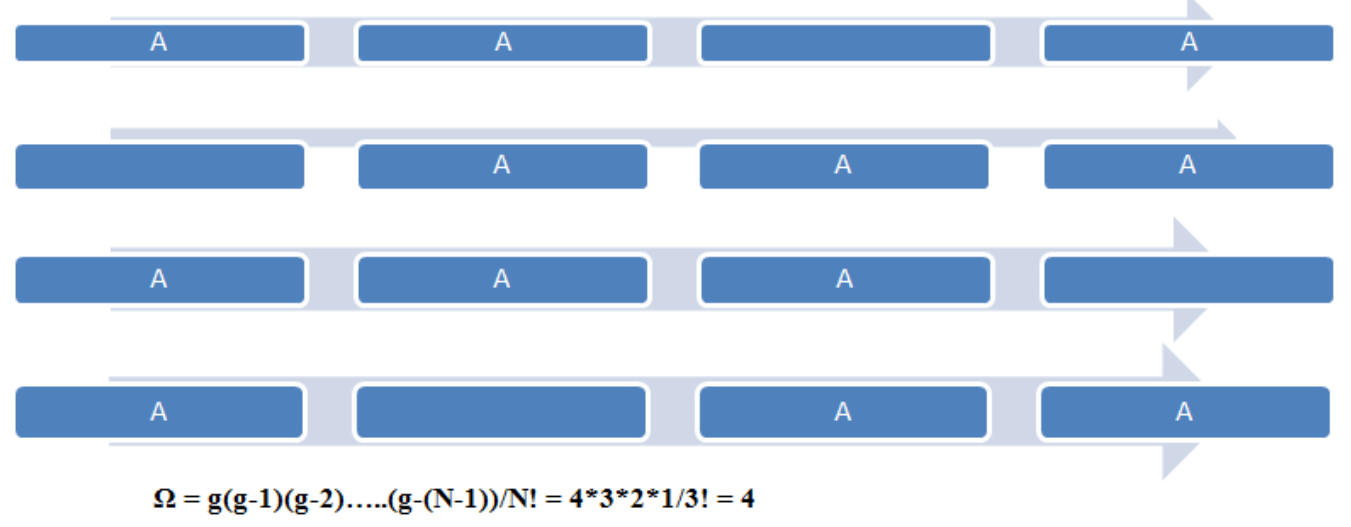




\section{Implementation using Computer Graphics}

Computer graphics is nothing but creation, storage and manipulation of pictures and drawings using digital computers. It enhances the power of communication between the computer and its user. The graphics facilities available on a computer system depend on its hardware and software. In graphics mode, the display screen is divided into a fine mesh of individual dots called pixels. Depending on the objects displayed, the corresponding pixels are either illuminated or darkened; forming the graphic objects on the screen [3].Turbo $\mathrm{C}$ is a high-level language which has been used to write programs for demonstrating the animation of the different examples. The $\mathrm{C}$ graphics library offers a large number of graphic functions that can be used to draw and animate the objects drawn.

The various microstates for example A1 (One distinguishable particle per cell) are as shown in Figs 1.1 to 1.9. (The Figures are screen shots and hence not very clear).

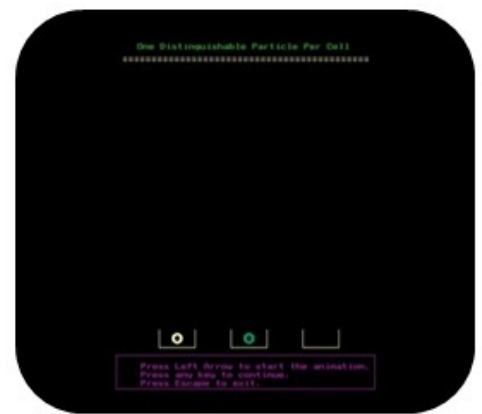

Fig1.1

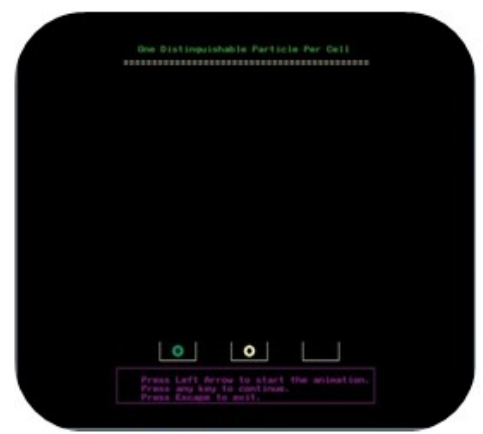

Fig 1.4

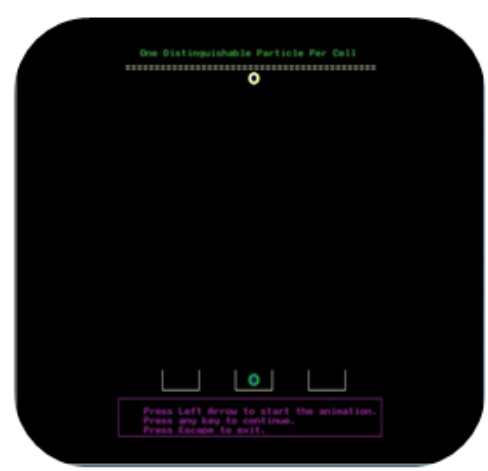

Fig 1.7

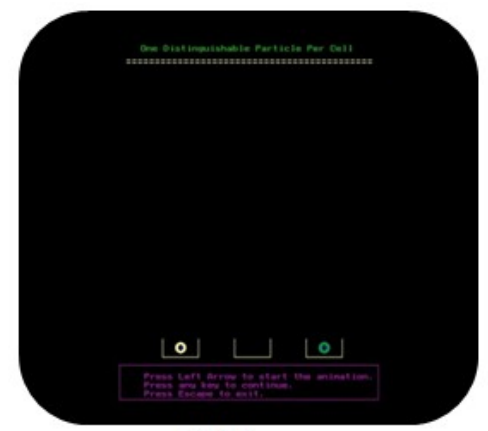

Fig1.2

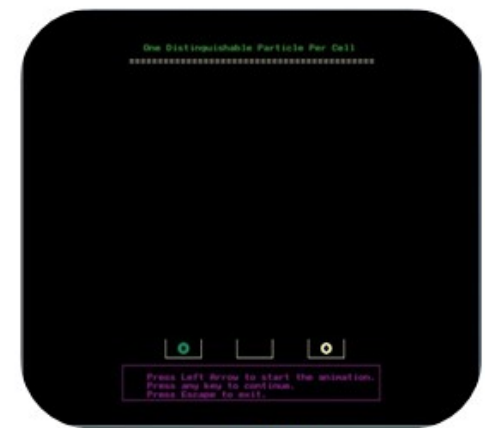

Fig 1.5

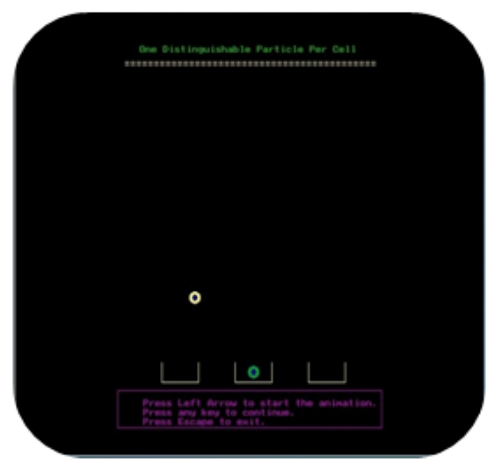

Fig 1.8

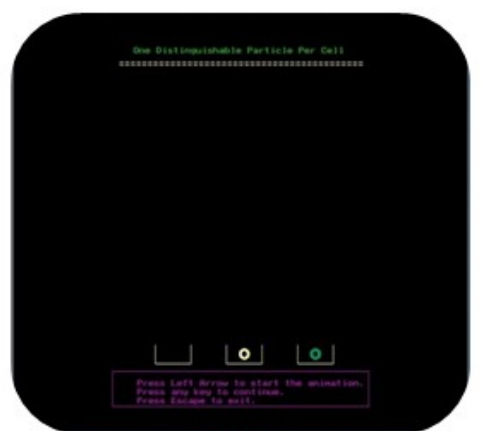

Fig 1.3

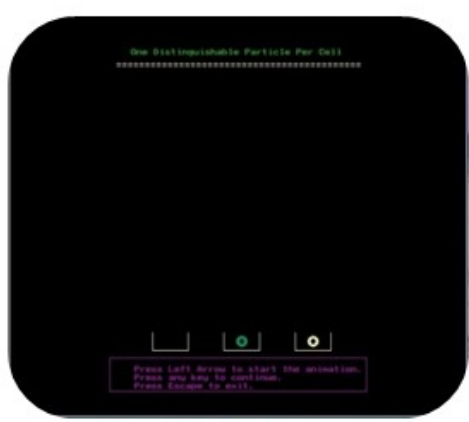

Fig1.6

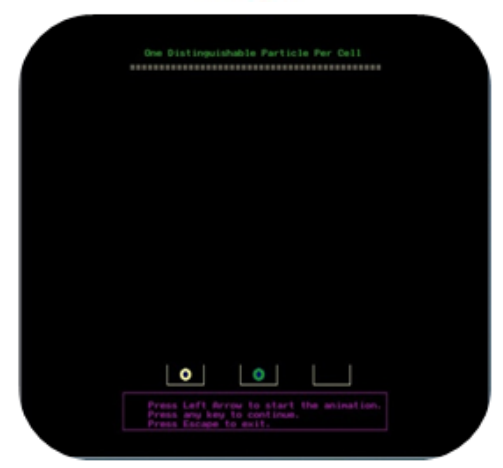

Fig 1.9

Figs 1.7, 1.8 and 1.9 show some intermediate positions during the movement of the yellow particle from cell 3 to cell1.

\section{Graphic Functions Used}

Some graphic functions used in the $\mathrm{C}$ programs are described below:

1) fillellipse(): This function draws and fills the circle/ellipse with the defined color.

2) setcolor(): This function sets the current drawing color.

3) setfillstyle(): This function is used to set the current fill pattern and color. Some fill styles available are Solid_fill, Line_fill, Slash_fill, Wide_dot fill etc. 
4) getimage(): This function saves a bit image of the specified region into memory. 5) putimage(): This function outputs a saved bit image on the screen.

Header files such as image.h and pos.h have been created to store the functions to draw images of the balls and their movement respectively. These header files have been included in the $\mathrm{C}$ programs to make it more structured.

\section{Conclusion}

The distinguishability and the number of particles per cell decide the statistics! Quantum or classical? Bose-Einstein or Maxwell-Boltzman? Fermi-Dirac or Maxwell-Boltzman? The animated demonstration for one of the cases is represented as pictures in this paper.

We have seen above, how difficult it is to understand the distribution, hence the statistics. This becomes easy by demonstrating using Computer graphics and animation. Learning and teaching, both become an entertainment. The events, scenes and the systems are brought to life.

\section{Acknowledgement}

I thank the Indian Association of Physics Teachers, for motivating me to learn Computational Physics and Dr M A Rama for teaching me C programming.

\section{Reference}

[1] Wikipedia

[2] F Rief, Statistical and Thermal Physics, Mc Graw Hill

[3] M A Rama, Computer Graphics, Subhas Stores 